\title{
A symbolic representation of the real Möbius group
}

\author{
Petr Kůrka ${ }^{1}$ \\ ${ }^{1}$ Center for Theoretical Study, Academy of Sciences and Charles University in \\ Prague, Jilská 1, CZ-11000 Praha 1, Czechia.
}

\begin{abstract}
We describe symbolic representations of the extended real line based on the dynamical systems consisting of Möbius transformations. The representations can be extended to the group of real Möbius transformations.
\end{abstract}

\section{Introduction}

A symbolic representation of real numbers can be regarded as a factor of a space of symbolic sequences. The binary representation of the unit interval $\mathbb{I}=[0,1]$ is the factor map $\Phi_{2}: \mathbb{Z}_{2}^{\mathbb{N}} \rightarrow \mathbb{I}$ defined by $\Phi_{2}(u)=\sum_{n=0}^{\infty} u_{n} 2^{-n-1}$. This can be obtained from a dynamical system $F: \mathbb{Z}_{2}^{*} \times \mathbb{I} \rightarrow \mathbb{I}$, where $\mathbb{Z}_{2}^{*}$ is the monoid of finite binary words. The dynamical system is generated by maps $F_{0}, F_{1}: \mathbb{I} \rightarrow \mathbb{I}$ given by $F_{i}(x)=(x+i) / 2$. For $u \in \mathbb{Z}_{2}^{k}, F_{u}$ is the composition $F_{u_{0}} \circ \cdots \circ F_{u_{k-1}}$. For an infinite word $u \in \mathbb{Z}_{2}^{\mathbb{N}}$, $\Phi_{2}(u)$ is the unique number contained in all $F_{u_{[0, k)}}(\mathbb{I})$, where $u_{[0, k)}$ is the prefix of $u$ of length $k$. This can be generalized to any contractive $A^{*}$-action $F: A^{*} \times X \rightarrow X$ (see Edgar [3], or Barnsley [1]). If all $F_{a}$ are contractions on a compact metric space $X$, then there exists a unique attractor $Y \subseteq X$ with $Y=\bigcup_{a \in A} F_{a}(Y)$, and a factor map $\Phi: A^{\mathbb{N}} \rightarrow Y$ such that $\{\Phi(u)\}=\cap_{k} F_{u_{[0, k)}}(X)$.

The standard binary or decadic representations are not very convenient, since continuous maps on $\mathbb{I}$ cannot be lifted to continuous maps on the symbolic space. For this reason, redundant number systems are used in computer arithmetics (see e.g. Frougny [4]). An example of a redundant number system is the base 10-system with digits $\{-5, \ldots, 5\}$ proposed by Cauchy [2]. To be able to perform continuous operations in the symbolic space, we need a topological extension property (see Theorem 1).

Theorem 1 For a Cantor space $X$ and a compact metric space $Y$ there exists a factor map $\Phi: X \rightarrow Y$ with the extension property. This means that for any continuous map $\varphi: X \rightarrow Y$ there exists a continuous map $F: X \rightarrow X$ such that $\Phi \circ F=\varphi$.
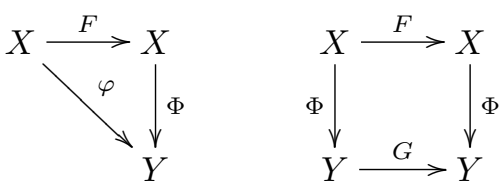

Thus, any continuous map $G: Y \rightarrow Y$ can be lifted to a continuous map $F: X \rightarrow X$ such that $\Phi \circ F=G \circ \Phi$. For a proof of Theorem 1 see Weihrauch [10] (Theorem 3.2.11, page 70) or Kůrka [6] (Theorem 3.8, page 110). 
The construction of a symbolic representation for the set $\mathbb{R}$ of real numbers poses another problem that $\mathbb{R}$ is not compact, so only a noncompact subset of the symbolic space could be used for the representation. A reasonable alternative is to construct a representation for a compactification of $\mathbb{R}$, e.g., for the extended real line $\overline{\mathbb{R}}=\mathbb{R} \cup\{\infty\}$. If we regard $\overline{\mathbb{R}}$ as a subspace of the complex sphere $\overline{\mathbb{C}}=\mathbb{C} \cup\{\infty\}$, a natural choice is a dynamical system whose generators are Möbius transformations, since these transformations are the only conformal isomorphisms of the complex sphere. The apparent problem in is that Möbius transformations are surjective, so the forward images of the space cannot converge to a point.

However, instead of convergence of sets, we can use convergence of measures, and inquire whether the images of the uniform measure converge to a point measure. This approach has an additional advantage, that finite numbers (i.e., finite words in the alphabet of digits) can be interpreted as imprecise numbers. The preciseness of a number increases with its length. As generators, we use local contractions to vertices of a regular polygon. The representation should satisfy some constraints, otherwise the contraction to a vertex would be cancelled by the contraction to the opposite vertex. These constraints define subshifts on the set of vertices of the polygon. We consider a family of walk subshifts parametrized by the speed of the walk around the circle, and show that symbolic representations of the extended real line can be based on them.

Moreover, we obtain also a symbolic representation of the group $\mathbb{M}_{\mathbb{R}}$ of real Möbius transformations. All compositions of our local contractions form a countable subgroup of $\mathbb{M}_{\mathbb{R}}$. We show that in some cases, this subgroup is dense in $\mathbb{M}_{\mathbb{R}}$. In this case, any transformation of $\mathbb{M}_{\mathbb{R}}$ can be represented as a converging sequence of elements of the subgroup.

\section{Möbius transformations}

A real orientation-preserving Möbius transformation (MT) is a selfmap of the extended real line $\overline{\mathbb{R}}:=\mathbb{R} \cup\{\infty\}$ of the form $M_{(a, b, c, d)}(x)=\frac{a x+b}{c x+d}$, where $a d-b c>0$. The space of these transformations can be parametrized with the use of the special linear group $\operatorname{SL}(2, \mathbb{R})=\left\{(a, b, c, d) \in \mathbb{R}^{4}: a d-b c=1\right\}$ with matrix multiplication and norm $\|(a, b, c, d)\|=\sqrt{a^{2}+b^{2}+c^{2}+d^{2}}$. We have the projective special linear group $\operatorname{PSL}(2, \mathbb{R})=\mathrm{SL}(2, \mathbb{R}) /\{\mathbf{1},-\mathbf{1}\}$, where $\mathbf{1}=(1,0,0,1)$ is the identity. With the quotient topology, $\operatorname{PSL}(2, \mathbb{R})$ is a continuous group which is in one-to-one correspondence with the real Möbius group $\mathbb{M}_{\mathbb{R}}=\left\{M_{(a, b, c, d)}: \overline{\mathbb{R}} \rightarrow \overline{\mathbb{R}}: a d-b c>0\right\}$.

Transformations $M_{(a, b, c, d)}$ act not only on $\overline{\mathbb{R}}$ but also on the upper half-plane $\mathbb{H}:=\{z \in \mathbb{C}: \Im(z)>0\}$, where they preserve the hyperbolic metric $d s=d z / \Im(z)$. $\mathbb{H}$ is conformally isomorphic to the unit $\operatorname{disc} \mathbb{D}=\{z \in \mathbb{C}:|z|<1\}$ via isomorphisms $\mathbf{d}: \mathbb{H} \rightarrow \mathbb{D}$ and $\mathbf{h}: \mathbb{D} \rightarrow \mathbb{H}$ given by $\mathbf{d}(z)=(z-i) /(-z-i)$, $\mathbf{h}(z)=(z-1) /(i z+i)$. Transformations $\mathbf{d}, \mathbf{h}$ extend to continuous maps on Euclidean closures $\overline{\mathbb{H}}=\{z \in \mathbb{C}: \Im(z) \geq 0\} \cup\{\infty\}$ and $\overline{\mathbb{D}}=\{z \in \mathbb{C}:|z| \leq 1\}$. On the closed unit disc we have disc Möbius transformations $\mathbf{M}_{(a, b, c, d)}=\mathbf{d} \circ M_{(a, b, c, d)} \circ \mathbf{h}$ given by

$$
\mathbf{M}_{(a, b, c, d)}(z)=\frac{[(d+a)+(b-c) i] z+(d-a)+(b+c) i}{[(d-a)-(b+c) i] z+(d+a)-(b-c) i} .
$$

The disc MT preserve the unit circle $\partial \mathbb{D}=\{z \in \mathbb{C}:|z|=1\}$. We parametrize the unit circle by $\mathbb{T}:=\mathbb{R} / 2 \pi \mathbb{Z}=[-\pi, \pi)$ with metric $d(t, s):=\min \{|s-t|, 2 \pi-|s-t|\}$, 
and we get mutually inverse transformations $x: \mathbb{T} \rightarrow \overline{\mathbb{R}}$ and $\mathbf{t}: \overline{\mathbb{R}} \rightarrow \mathbb{T}$ given by $\boldsymbol{\chi}(t)=\mathbf{h}\left(e^{i t}\right)=\tan \frac{t}{2}, \mathbf{t}(x)=\arg \mathbf{d}(x)=2 \arctan x$. We have circle Möbius transformations $\mathrm{M}_{(a, b, c, d)}=\mathbf{t} \circ M_{(a, b, c, d)} \circ \boldsymbol{x}: \mathbb{T} \rightarrow \mathbb{T}$. Alternatively, we can view them as increasing continuous functions $\mathrm{M}: \mathbb{R} \rightarrow \mathbb{R}$ with $\mathrm{M}(t+2 \pi)=\mathrm{M}(t)+2 \pi$. Denote by $\mathbb{M}_{\mathbb{D}}$ the group of disc MT and by $\mathbb{M}_{\mathbb{T}}$ the group of circle MT. The continuous groups $\mathbb{M}_{\mathbb{R}}, \mathbb{M}_{\mathbb{D}}$, and $\mathbb{M}_{\mathbb{T}}$ are all homeomorphic to $\operatorname{PSL}(2, \mathbb{R})$.

The trace of a MT is not well-defined but the square of the trace is well defined by $\operatorname{tr}^{2}\left(M_{(a, b, c, d)}\right):=(a+d)^{2} /(a d-b c)$. A transformation $\mathbf{M} \in \mathbb{M}_{\mathbb{D}}$ is elliptic if $\operatorname{tr}^{2}(\mathbf{M})<4$, parabolic if $\operatorname{tr}^{2}(\mathbf{M})=4$, and hyperbolic if $\operatorname{tr}^{2}(\mathbf{M})>4$. A point $z \in \overline{\mathbb{C}}$ is a fixed point of $\mathbf{M}$ if $\mathbf{M}(z)=z$, and it is stable if $\left|\mathbf{M}^{\prime}(z)\right| \leq 1$. A hyperbolic MT has two fixed points in $\partial \mathbb{D}$ and exactly one of them is stable. A parabolic MT has a unique stable fixed point (this is not stability in dynamical sense), which is in $\partial \mathbb{D}$. An elliptic MT has two fixed points and exactly one of them is in $\mathbb{D}$.

Disc MT preserve hyperbolic metric $d s=d z /\left(1-|z|^{2}\right)$ on $\mathbb{D}$. A disc MT $\mathbf{M} \in \mathbb{M}_{\mathbb{D}}$ is determined by its value $\mathbf{M}(0)$ and the unit tangent vector $\mathbf{M}^{\bullet}(0)$, where $\mathbf{M}^{\bullet}(z):=\mathbf{M}^{\prime}(z) /\left|\mathbf{M}^{\prime}(z)\right|$ for $z \in \mathbb{D}$. Define the unit tangent bundle of $\mathbb{D}$ as $T \mathbb{D}=\mathbb{D} \times \partial \mathbb{D}$. There exists a homeomorphism $\mathcal{H}: \mathbb{M}_{\mathbb{D}} \rightarrow T \mathbb{D}$ given by $\mathcal{H}(\mathbf{M})=\left(\mathbf{M}(0), \mathbf{M}^{\bullet}(0)\right)$ (see Katok [5], Thm 2.1.1). A transformation $\mathbf{M} \in \mathbb{M}_{\mathbb{D}}$ acts on $T \mathbb{D}$ by $\mathbf{M}(z, \xi)=\left(\mathbf{M}(z), \mathbf{M}^{\bullet}(z) \cdot \xi\right)$ and preserves the Riemannian metric

$$
d s^{2}=\frac{d x^{2}+d y^{2}}{\left(1-x^{2}-y^{2}\right)^{2}}+\frac{d \xi^{2}}{4 \pi^{2}}, \quad(x+i y, \xi) \in T \mathbb{D}
$$

The group operation of $\mathbb{M}_{\mathbb{D}}$ is preserved by $\mathcal{H}$, i.e., $\mathcal{H}(\mathbf{M} \circ \mathbf{N})=\mathbf{M}(\mathcal{H}(\mathbf{N}))$. This means that $T \mathbb{D}$ is a continuous group homeomorphic to $\mathbb{M}_{\mathbb{D}}$. The action of $\mathbf{M}$ can be continuously extended on the Euclidean closure $\overline{T \mathbb{D}}=\overline{\mathbb{D}} \times \partial \mathbb{D}$ of the unit tangent bundle by the same formula $\mathbf{M}(z, \xi)=\left(\mathbf{M}(z), \mathbf{M}^{\bullet}(z) \cdot \xi\right)$. We have

$$
\begin{aligned}
& \mathbf{M}_{(a, b, c, d)}(0)=\frac{\left(c^{2}+d^{2}-a^{2}-b^{2}\right)+2(a c+b d) i}{(d+a)^{2}+(b-c)^{2}}, \\
& \mathbf{M}_{(a, b, c, d)}^{\bullet}(0)=\frac{\left((d+a)^{2}-(b-c)^{2}\right)+2(d+a)(b-c) i}{(d+a)^{2}+(b-c)^{2}} .
\end{aligned}
$$

Denote by $\mathrm{C}_{r}(t)=2 \arctan \left(r^{2} \tan \frac{t}{2}\right)$ the contraction to 0 with quotient $r<1$ which is the circle Möbius transformation corresponding to the contraction $C_{r}(x)=r^{2} x$ of the real line. We have

$$
C_{r}^{\prime}(t)=r^{2} \frac{1+\chi^{2}(t)}{1+r^{4} \chi^{2}(t)}, \quad\left|C_{r}^{\prime}(t)\right| \leq 1 \Longleftrightarrow|\boldsymbol{x}(t)| \leq \frac{1}{r} \Longleftrightarrow|t| \leq \pi-\alpha_{r},
$$

where $\alpha_{r}=2 \arctan r<\pi / 2$. Denote by $U_{0}=\left[\alpha_{r}-\pi, \pi-\alpha_{r}\right]$ the contraction interval of $C_{r}$ and by $V_{0}=\left[-\alpha_{r}, \alpha_{r}\right]$ the expansion interval of $\mathrm{C}_{r}^{-1}=\mathrm{C}_{\frac{1}{r}}$ respectively. Note that $\mathrm{C}_{r}\left(U_{0}\right)=V_{0} \subset U_{0}$, so $\mathrm{C}_{r}\left(\pi-\alpha_{r}\right)=\alpha_{r}$. Denote by $R_{\beta}(x)=\left(\cos \frac{\beta}{2} \cdot x+\sin \frac{\beta}{2}\right) /\left(-\sin \frac{\beta}{2} \cdot x+\cos \frac{\beta}{2}\right)$ the real MT corresponding to the rotation $\mathrm{R}_{\beta}(t)=t+\beta$. We consider also contractions $\mathrm{C}_{r, \beta}=\mathrm{R}_{\beta} \circ \mathrm{C}_{r} \circ \mathrm{R}_{-\beta}$ to points $\beta \in \mathbb{T}$.

Lemma 2 For $0<r<1$ there exists an increasing continuous function $\psi_{r}$ : $\left[0,2\left(\pi-\alpha_{r}\right)\right] \rightarrow\left[0,2 \alpha_{r}\right]$ such that $\psi_{r}(0)=0,0<\psi_{r}(t)<t$ for $t>0$ and $\left|\mathrm{C}_{r}(W)\right| \leq \psi_{r}(|W|)$ for each interval $W \subseteq U_{0}$.

Here $|W|$ is the length of the interval $W$. The proof follows from the fact that $C_{r}^{\prime}(t)<1$ for each $t \in \operatorname{int}\left(U_{0}\right)$. 


\section{Measures}

The value $\mathbf{M}(0)$ of a disc MT can be interpreted as a mean of a probability distribution. Given a compact metric space $X$, we have the space $\mathfrak{M}(X)$ of Borel probability measures with weak* topology, i.e., $\lim _{n \rightarrow \infty} \mu_{n}=\mu$ iff $\lim _{n \rightarrow \infty} \int f d \mu_{n}=\int f d \mu$ for each continuous function $f$. A continuous function $F: X \rightarrow Y$ extends to a continuous function $F: \mathfrak{M}(X) \rightarrow \mathfrak{M}(Y)$ by $(F \mu)(U)=\mu\left(F^{-1}(U)\right)$. Denote by $\delta_{x}$ the Dirac point measure concentrated on $x$, i.e., $\delta_{x}(U)=1$ iff $x \in U$. Measures on $\mathbb{T}$ which are absolutely continuous with respect to the Lebesgue measure have densities, which are nonnegative functions with unit integral. In particular the uniform Lebesgue measure $\ell$ on $\mathbb{T}$ has the constant probability density $h(t)=1 / 2 \pi$. Since $\mathrm{M}_{(a, b, c, d)}^{-1}=\mathrm{M}_{(d,-b,-c, a)}$, the probability density of $\mathrm{M}_{(a, b, c, d)} \ell$ is

$$
\begin{aligned}
h_{(a, b, c, d)}(t) & =h\left(\mathrm{M}_{(a, b, c, d)}^{-1}(t)\right) \cdot\left(\mathrm{M}_{(a, b, c, d)}^{-1}\right)^{\prime}(t) \\
& =\frac{1}{2 \pi} \cdot \frac{(a d-b c)\left(1+\boldsymbol{x}^{2}(t)\right)}{(d \cdot \boldsymbol{x}(t)-b)^{2}+(c \cdot \boldsymbol{x}(t)-a)^{2}} .
\end{aligned}
$$

A measure $\mu \in \mathfrak{M}(\mathbb{T})$ can be characterized by its mean $\mathcal{E}(\mu):=\int_{\mathbb{T}} d \mu$ which is a complex number in the closed unit disc $\overline{\mathbb{D}}$. For a measure with density $h$ we get $\mathcal{E}(h)=\int_{-\pi}^{\pi} h(t) e^{i t} d t \in \mathbb{D}$. For a point measure we have $\mathcal{E}\left(\delta_{t}\right)=e^{i t} \in \partial \mathbb{D}$.

Proposition 3 Let $\mathbf{M} \in \mathbb{M}_{\mathbb{T}}$ be a circle $M T$ and $\mathbf{M} \in \mathbb{M}_{\mathbb{D}}$ the corresponding disc $M T$. Then $\mathcal{E}(\mathrm{M} \ell)=\mathbf{M}(0)$.

Proof: For $w \in \partial \mathbb{D}$ we have $\arg w=\frac{1}{i} \log w$. We use the substitutions $z=e^{i t}$ and $w=\mathbf{M}^{-1}(z)$ to get

$$
\begin{aligned}
\mathcal{E}(\mathrm{M} \ell) & =\frac{1}{2 \pi} \int_{-\pi}^{\pi}\left(\mathrm{M}^{-1}\right)^{\prime}(t) \cdot e^{i t} d t=\frac{1}{2 \pi} \int_{-\pi}^{\pi}\left(\mathbf{t} \circ M^{-1} \circ \mathbf{h}\right)^{\prime}\left(e^{i t}\right) \cdot i e^{2 i t} d t \\
& =\frac{1}{2 \pi} \int_{\mathbb{T}}\left(\mathbf{t} \circ M^{-1} \circ \mathbf{h}\right)^{\prime}(z) \cdot z d z \\
& =\frac{1}{2 \pi} \int_{\mathbb{T}} \frac{1}{i} \log ^{\prime}\left(\mathbf{M}^{-1}(z)\right) \cdot\left(\mathbf{M}^{-1}\right)^{\prime}(z) \cdot z d z \\
& =\frac{1}{2 \pi i} \int_{\mathbb{T}} \frac{\left(\mathbf{M}^{-1}\right)^{\prime}(z)}{\mathbf{M}^{-1}(z)} \cdot z d z=\frac{1}{2 \pi i} \int_{\mathbb{T}} \frac{\mathbf{M}(w) d w}{w}=\mathbf{M}(0) .
\end{aligned}
$$

The last equality is due to the Cauchy integral formula. Indeed, the last few lines follow from a variant of the "argument principle". More elementarily we get (using the substitution $t=\mathbf{t}(x)$ )

$$
\begin{aligned}
\mathcal{E}\left(\mathrm{M}_{(a, b, c, d)} \ell\right) & =\int_{-\infty}^{\infty} h_{(a, b, c, d)}(\mathbf{t}(x)) \cdot \frac{1-x^{2}+2 i x}{1+x^{2}} \cdot \frac{2 d x}{1+x^{2}} \\
& =\int_{-\infty}^{+\infty} \frac{(a d-b c)\left[1-x^{2}+2 i x\right] d x}{\pi\left[(d \cdot x-b)^{2}+(c \cdot x-a)^{2}\right]\left(1+x^{2}\right)} \\
& =\frac{\left(c^{2}+d^{2}-a^{2}-b^{2}\right)+2(a c+b d) i}{(a+d)^{2}+(b-c)^{2}}=\mathbf{M}_{(a, b, c, d)}(0) .
\end{aligned}
$$

In the sequel we use the following lemma whose proof follows from the fact that a density $h_{(a, b, c, d)}: \mathbb{T} \rightarrow[0, \infty)$ has only one local maximum in $\mathbb{T}$. 
Lemma 4 Let $\left(\mathrm{M}_{n}: \mathbb{T} \rightarrow \mathbb{T}\right)_{n \geq 0}$ be a sequence of circle $M T$. Assume that there exists $t \in \mathbb{T}$ and $c>0$ such that for each interval $I \ni t$ we have $\liminf _{n \rightarrow \infty}\left(\mathrm{M}_{n} \ell\right)(I)>c$. Then $\lim _{n \rightarrow \infty}\left(\mathrm{M}_{n} \ell\right)(I)=1$ and $\lim _{n \rightarrow \infty} \mathrm{M}_{n} \ell=\delta_{t}$.

\section{Subshifts and dynamical systems}

For a finite alphabet $A$, denote by $A^{*}:=\bigcup_{m \geq 0} A^{m}$ the set of words over $A$ and by $A^{+}:=\bigcup_{m>0} A^{m}$ the set of nonempty words. The length of a word $u=u_{0} \ldots u_{m-1} \in$ $A^{m}$ is denoted by $|u|:=m$ and the word of zero length is $\lambda$. We say that $u \in A^{*}$ is a subword of $v \in A^{*}(u \sqsubseteq v)$, if there exists $k$ such that $v_{k+i}=u_{i}$ for $i<|u|$. We denote by $u_{[i, j)}=u_{i} \ldots u_{j-1}$ and $u_{[i, j]}=u_{i} \ldots u_{j}$ subwords of $u$ associated to intervals. With the operation of concatenation and empty word $\lambda, A^{*}$ is the free monoid over $A$.

We denote by $A^{\mathbb{N}}$ the Cantor space of infinite sequences of letters of $A$ equipped with the metric $d(x, y):=2^{-k}$, where $k=\min \left\{i \geq 0: x_{i} \neq y_{i}\right\}$. The shift map $\sigma: A^{\mathbb{N}} \rightarrow A^{\mathbb{N}}$ is defined by $\sigma(u)_{i}=u_{i+1}$. A subshift is a nonempty subset $\Sigma \subseteq A^{\mathbb{N}}$, which is closed and $\sigma$-invariant, i.e., $\sigma(\Sigma) \subseteq \Sigma$. For a subshift $\Sigma$ there exists a set $D \subseteq A^{*}$ of forbidden words such that $\Sigma=\mathcal{S}_{D}:=\left\{x \in A^{\mathbb{N}}: \forall u \sqsubseteq x, u \notin D\right\}$. A subshift is of finite type (SFT), if there exists a finite set $D \subset A^{*}$ such that $\Sigma=\mathcal{S}_{D}$. The order $\mathfrak{o}(\Sigma)$ of a SFT is the minimal integer $k>0$ such that $\Sigma=\mathcal{S}_{D}$ for some $D \subseteq A^{k}$. A subshift is uniquely determined by its language $\mathcal{L}(\Sigma):=\left\{u \in A^{*}: \exists x \in \Sigma, u \sqsubseteq x\right\}$ (see e.g., Lind and Marcus [8]). The cylinder of a word $u \in \mathcal{L}(\Sigma)$ is $[u]:=\left\{v \in \Sigma: v_{[0,|u|)}=u\right\}$. Given $v \in A^{k}, v^{\infty} \in A^{\mathbb{N}}$ is defined by $\left(v^{\infty}\right)_{k m+j}=v_{j}$.

By a dynamical system we mean an $A^{*}$-action over a compact metric space $X$, i.e., a continuous map $F: A^{*} \times X \rightarrow X$ satisfying $F_{\lambda}=\operatorname{Id}_{X}$ and $F_{u v}=F_{u} \circ F_{v}$, (the discrete topology is assumed on $A^{*}$ ). The action is given by generators $\left(F_{a}: X \rightarrow X\right)_{a \in A}$. As alphabets we use groups $\mathbb{Z}_{n}=\mathbb{Z} / n \mathbb{Z}=\{0,1, \ldots, n-1\}$ of integers modulo $n$ with circle distance defined by $d_{n}(a, b)=\min \{|a-b|, n-|a-b|\}$. By an interval of $\mathbb{Z}_{n}$ we mean either $[a, b]:=\left[c \in \mathbb{Z}_{n}: a \leq c \leq b\right]$ if $a \leq b$ or $[a, b]:=\left[c \in \mathbb{Z}_{n}: a \leq c\right.$ or $\left.c \leq b\right\}$ if $a>b$. Similarly we understand intervals in $\mathbb{T}=[-\pi, \pi)$.

Definition $5 A$ hyperbolic number system with arity $n>2$ and quotient $0<$ $r<1$ is a $\mathbb{Z}_{n}^{*}$-action $\mathrm{F}: \mathbb{Z}_{n}^{*} \times \mathbb{T} \rightarrow \mathbb{T}$, where $\mathrm{F}_{a}=\mathrm{C}_{r, \frac{2 \pi a}{n}}$ for $a \in \mathbb{Z}_{n}$. Denote by $\alpha_{r}=2 \arctan r, U_{a}=\left[-\pi+\alpha_{r}+\frac{2 \pi a}{n}, \pi-\alpha_{r}+\frac{2 \pi a}{n}\right], V_{a}=\left[-\alpha_{r}+\frac{2 \pi a}{n}, \alpha_{r}+\frac{2 \pi a}{n}\right]$ the contraction intervals of $\mathrm{F}_{a}$ and the expansion intervals of $\mathrm{F}_{a}^{-1}$ respectively. The convergence space $\mathbb{X}_{n, r} \subseteq \mathbb{Z}_{n}^{\mathbb{N}}$ and the map $\Phi_{n, r}: \mathbb{X}_{n, r} \rightarrow \mathbb{T}$ are defined by

$$
\mathbb{X}_{n, r}:=\left\{u \in \mathbb{Z}_{n}^{\mathbb{N}}:\left|\lim _{j \rightarrow \infty} \mathbf{F}_{u_{[0, j)}}(0)\right|=1\right\}, \quad \Phi_{n, r}(u)=\arg \lim _{j \rightarrow \infty} \mathbf{F}_{u_{[0, j)}}(0)
$$

The space $\mathbb{X}_{n, r}$ is dense in $\mathbb{Z}_{n}^{\mathbb{N}}$ and it is closed with respect to finite modifications. If $u \in \mathbb{Z}_{n}^{\mathbb{N}}$ and $v \in \mathbb{Z}_{n}^{*}$, then $u \in \mathbb{X}_{n, r}$ iff $v u \in \mathbb{X}_{n, r}$. In this case $\Phi_{n, r}(v u)=\mathrm{F}_{v}\left(\Phi_{n, r}(u)\right)$.

Proposition 6 Let $\mathrm{F}: \mathbb{Z}_{n}^{*} \times \mathbb{T} \rightarrow \mathbb{T}$ be a hyperbolic number system and $u \in \mathbb{Z}_{n}^{\mathbb{N}}$. Then

$$
\lim _{j \rightarrow \infty} \mathrm{F}_{u_{[0, j)}} \ell=\delta_{t} \Longleftrightarrow \lim _{j \rightarrow \infty} \mathbf{F}_{u_{[0, j)}}(0)=e^{i t}
$$

The proof follows from Proposition 3.

Proposition $\mathbf{7}$ For $v \in \mathbb{Z}_{n}^{+}$we have $v^{\infty} \in \mathbb{X}_{n, r}$ iff $\mathbf{F}_{v}$ is not elliptic. In this case $\Phi_{n, r}\left(v^{\infty}\right)$ is the stable fixed point of $\mathbf{F}_{v}$. 
Proof: An elliptic transformation has an invariant circle which passes through 0 . All $\mathbf{F}_{v^{k}}(0)$ lie on this circle and cannot converge to any point in $\partial \mathbb{D}$. If $\mathbf{F}_{v}$ is hyperbolic or parabolic with stable fixed point $e^{i t}$, then $e^{i t}$ attracts all points of $\mathbb{D}$.

Proposition 8 For $u \in \mathbb{Z}_{n}^{*}$ and $a \in \mathbb{Z}_{n}$ denote by $\gamma_{u, a}$ the unique hyperbolic geodesic which starts at $\mathbf{F}_{u}(0)$ and passes through $\mathbf{F}_{u a}(0)$. Then all points $\left\{\mathbf{F}_{u a^{j}}(0): j \geq 0\right\}$ lie on $\gamma_{u, a}$. The angle of geodesics $\gamma_{u, a}$ and $\gamma_{u, b}$ at $\mathbf{F}_{u}(0)$ is $2 \pi(b-a) / n$.

Proof: For $a \in \mathbb{Z}_{n}$, the stable and unstable fixed points of $\mathbf{F}_{a}$ are opposite and the diameter which joins them is a geodesic which passes through 0 , so the statement is true when $u=\lambda$. If $u$ is arbitrary, then the $\mathbf{F}_{u}$-image of $\gamma_{\lambda, a}$ is a geodesic and contains points $\mathbf{F}_{u a^{j}}(0)=\mathbf{F}_{u}\left(\mathbf{F}_{a^{j}}(0)\right)$, so $\mathbf{F}_{u}\left(\gamma_{\lambda, a}\right)=\gamma_{u, a}$. The angle of $\gamma_{u, a}$ and $\gamma_{u, b}$ is the same as the angle between $\gamma_{\lambda, a}$ and $\gamma_{\lambda, b}$, which is $2 \pi(b-a) / n$ (see Figure 1).

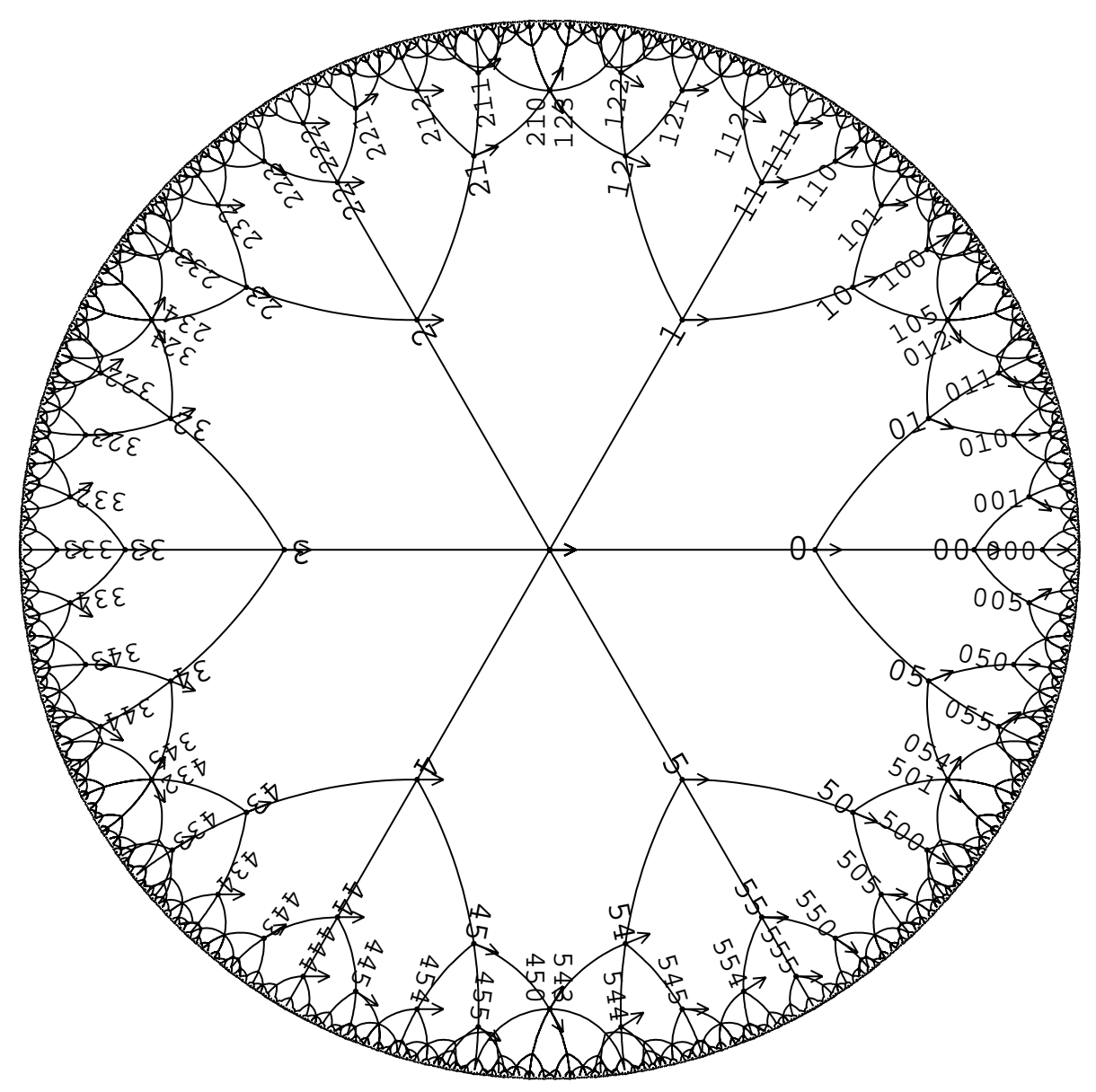

Figure 1. Means and unit tangent vectors of hyperbolic numbers for $n=6$, $r=\mathfrak{q}_{6,1}=1 / \sqrt{3}$ 


\section{Walk subshifts}

Definition 9 For $n \geq 4$ and $1 \leq k \leq \frac{n}{2}-1$ define the walk subshift $\mathbb{W}_{n, k} \subseteq \mathbb{Z}_{n}^{\mathbb{N}}$ as the SFT of order 2 with forbidden words $D_{n, k}=\left\{a b \in \mathbb{Z}_{n}^{2}: d_{n}(a, b)>k\right\}$. For $u \in \mathbb{Z}_{n}^{j}$ set $V_{u}=\mathrm{F}_{u}\left(U_{u_{j-1}}\right)$. The expansion and contraction quotients are defined by

$$
\mathfrak{p}_{n, k}:=\sqrt{\frac{\sin \frac{(k+1) \pi}{n}-\sin \frac{k \pi}{n}}{\sin \frac{(k+1) \pi}{n}+\sin \frac{k \pi}{n}}}, \quad \mathfrak{q}_{n, k}:=\sqrt{\frac{1-\sin \frac{k \pi}{n}}{1+\sin \frac{k \pi}{n}}} .
$$

Thus if $k=1$, the only allowed transitions are $a \rightarrow(a-1), a \rightarrow a$, and $a \rightarrow(a+1)$ to neighbouring letters. The definition of $V_{u}$ extends the previous use since $V_{a}=\mathrm{F}_{a}\left(U_{a}\right)$ for $a \in \mathbb{Z}_{n}$. Note that $\mathfrak{p}_{n, k} \leq \mathfrak{q}_{n, k}$ (see Table 1). We are going to prove that hyperbolic number systems work for walk subshifts $\mathbb{W}_{n, k}$ and quotients $\mathfrak{p}_{n, k} \leq r \leq \mathfrak{q}_{n, k}$.

Theorem 10 Let $1 \leq k \leq \frac{n}{2}-1$ and let $\mathrm{F}: \mathbb{Z}_{n}^{*} \times \mathbb{T} \rightarrow \mathbb{T}$ be a hyperbolic number system with quotient $0<r \leq \mathfrak{q}_{n, k}$. Then $\mathbb{W}_{n, k} \subseteq \mathbb{X}_{n, r}$ and $\Phi_{n, k}: \mathbb{W}_{n, k} \rightarrow \mathbb{T}$ is continuous. For any $u \in \mathbb{W}_{n, k}$ we have $V_{u_{[0, j)}} \subseteq V_{u_{[0, j-1)}}$ and $\bigcap_{j>0} V_{u_{[0, j)}}=\left\{\Phi_{n, k}(u)\right\}$.

Proof: We show first that $\mathrm{F}_{a}\left(U_{a}\right) \subseteq \bigcap_{b=-k}^{k} U_{a+b}$ for each $a \in \mathbb{Z}_{n}$. Because of the symmetries, the condition is satisfied iff it is satisfied for $a=0$. This gives $\left[-\alpha_{r}, \alpha_{r}\right] \subseteq\left[-\pi+\alpha_{r}+\frac{2 \pi k}{n}, \pi-\alpha_{r}-\frac{2 \pi k}{n}\right]$, i.e., $\alpha_{r} \leq \frac{\pi}{2}-\frac{k \pi}{n}$, or $r \leq \tan \left(\frac{\pi}{4}-\frac{k \pi}{2 n}\right)=\mathfrak{q}_{n, k}$. Let $u \in \mathbb{W}_{n, k}$ and $j>0$. Since $u_{[j-2, j-1]} \in \mathcal{L}\left(\mathbb{W}_{n, k}\right)$, we have $\mathbf{F}_{u_{j-1}}\left(U_{u_{j-1}}\right) \subseteq U_{u_{j-2}}$. Applying $\mathrm{F}_{u_{[0, j-2]}}$ we get $V_{u_{[0, j)}}=\mathrm{F}_{u_{[0, j-2]}}\left(\mathrm{F}_{u_{j-1}}\left(U_{u_{j-1}}\right)\right) \subseteq \mathrm{F}_{u_{[0, j-2]}}\left(U_{u_{j-2}}\right)=V_{u_{[0, j-1)}}$. Using the contraction function $\psi_{r}$ of Lemma 2 , we get

$$
\left|V_{u_{[0, j)}}\right| \leq \psi_{r}\left(\left|\mathrm{~F}_{u_{[1, j)}}\left(U_{u_{j-1}}\right)\right|\right) \leq \psi_{r}^{2}\left(\left|\mathrm{~F}_{u_{[2, j)}}\left(U_{u_{j-1}}\right)\right|\right) \leq \cdots \leq \psi_{r}^{k}\left(\left|U_{u_{j-1}}\right|\right) .
$$

Since the only fixed point of $\psi_{r}$ is 0 , we have $\lim _{j \rightarrow \infty}\left|V_{\left.u_{[0, j}\right)}\right|=0$, so $\bigcap_{j} V_{u_{[0, j)}}$ is a singleton, and we denote its unique element by $\Psi(u)$. Clearly $\Psi: \mathbb{W}_{n, k} \rightarrow \mathbb{T}$ is continuous. Since $\left(\mathrm{F}_{u_{[0, j)}} \ell\right)\left(V_{j}\right)=\ell\left(\left(\mathrm{F}_{\left.u_{[0, j}\right)}\right)^{-1} \mathrm{~F}_{u_{[0, j)}}\left(U_{u_{j-1}}\right)\right)=\ell\left(U_{u_{j-1}}\right)=2(\pi-\alpha)$, we have $\lim _{n \rightarrow \infty} \mathrm{F}_{u_{[0, j)}} \ell=\delta_{\Psi(u)}$ by Lemma 4 , so $u \in \mathbb{X}_{n, r}$ and $\Psi(u)=\Phi_{n, k}(u)$ by Proposition 6 .

For $a, b \in \mathbb{Z}_{n}$ write $a \prec b$ if $0 \leq b-a<n / 2$. This is an order on each interval of $\mathbb{Z}_{n}$ whose length does not exceed $n / 2$. Recall that + and - are group operations modulo $n$, so $(n-1) \prec 0$. We extend the relation $\prec$ to $\mathbb{W}_{n, k}$ lexicographically. We have $u \prec v$ if there exists $j$ such that $u_{[0, j)}=v_{[0, j)}$ and $u_{j} \prec v_{j}$. Given $u \in \mathcal{L}\left(\mathbb{W}_{n, k}\right)$, the minimal and maximal elements $l(u)$ and $r(u)$ of $[u]$ are well defined by

$$
l(u)_{i}=\left\{\begin{array}{ll}
u_{i} & \text { for } i<|u| \\
u_{i-1}-k & \text { for } i \geq|u|
\end{array} \quad, \quad r(u)_{i}= \begin{cases}u_{i} & \text { for } i<|u| \\
u_{i-1}+k & \text { for } i \geq|u|\end{cases}\right.
$$

Similarly, for $t, s \in \mathbb{T}$ write $t \prec s$ if $0<s-t<\pi$, where - is the group subtraction modulo $2 \pi$.

Theorem 11 Let $1 \leq k \leq \frac{n}{2}-1$ and let $\mathrm{F}: \mathbb{Z}_{n}^{*} \times \mathbb{T} \rightarrow \mathbb{T}$ be a hyperbolic number system with quotient $r>\mathfrak{q}_{n, k}$. Then $\mathbb{W}_{n, k} \not \mathbb{\mathbb { X } _ { n , r }}$. 


\begin{tabular}{|l|l|l|l|l|l|l|l|l|l|}
\hline$n$ & 4 & 5 & 6 & 7 & 8 & 9 & 10 & 11 & 12 \\
\hline $\mathfrak{p}_{n, 1}$ & 0.414 & 0.486 & 0.518 & 0.535 & 0.546 & 0.553 & 0.558 & 0.561 & 0.564 \\
$\mathfrak{q}_{n, 1}$ & 0.414 & 0.510 & 0.577 & 0.628 & 0.668 & 0.700 & 0.727 & 0.749 & 0.767 \\
\hline $\mathfrak{p}_{n, 2}$ & & & 0.268 & 0.332 & 0.365 & 0.385 & 0.398 & 0.407 & 0.414 \\
$\mathfrak{q}_{n, 2}$ & & & 0.268 & 0.350 & 0.414 & 0.466 & 0.510 & 0.546 & 0.577 \\
\hline
\end{tabular}

Table 1. Expansion and contraction quotients

Proof: Let $u=r(0) \in \mathbb{W}_{n, k}$ be the maximal element of the cylinder [0]. This is a periodic sequence $u=v^{\infty}$, where $v:=u_{[0, n)}$. We show that for $r>\mathfrak{q}_{n, k}, \mathbf{F}_{v}$ is an elliptic transformation, so $u \notin \mathbb{X}_{n, r}$ by Proposition 7 . We have the real MT $C_{r}(x)=r^{2} x$, the rotation MT $R_{j}$ and their product with matrices

$$
C_{r}=\left[\begin{array}{ll}
r & 0 \\
0 & \frac{1}{r}
\end{array}\right], \quad R_{j}=\left[\begin{array}{rr}
\cos \frac{j \pi}{n} & \sin \frac{j \pi}{n} \\
-\sin \frac{j \pi}{n} & \cos \frac{j \pi}{n}
\end{array}\right], \quad C_{r} R_{j}=\left[\begin{array}{rr}
r \cos \frac{j \pi}{n} & r \sin \frac{j \pi}{n} \\
-\frac{1}{r} \sin \frac{j \pi}{n} & \frac{1}{r} \cos \frac{j \pi}{n}
\end{array}\right]
$$

We have $F_{v}=C_{r}\left(R_{k} C_{r} R_{-k}\right)\left(R_{2 k} C_{r} R_{-2 k}\right) \cdots\left(R_{(n-1) k} C_{r} R_{-(n-1) k}\right)=\left(C_{r} R_{k}\right)^{n} \cdot C_{-k n}$. Since $C_{-k n}$ is either $\mathbf{1}$ (identity) or $\mathbf{- 1}$, we get $F_{v}=\left(C_{r} R_{k}\right)^{n}$. If $r>\mathfrak{q}_{n, k}$, then $\operatorname{tr}^{2}\left(C_{r} R_{k}\right)<4$, so $C_{r} R_{k}$ is elliptic and $F_{v}$ is elliptic as well.

Proposition 12 Let $1 \leq k \leq \frac{n}{2}-1$ and let $\mathrm{F}: \mathbb{Z}_{n}^{*} \times \mathbb{T} \rightarrow \mathbb{T}$ be a hyperbolic number system with $r \leq \mathfrak{q}_{n, k}$. For $u \in \mathcal{L}\left(\mathbb{W}_{n, k}\right)$ set $W_{u}:=\left[\Phi_{n, k}(l(u)), \Phi_{n, k}(r(u))\right] \subset \mathbb{T}$. Then $W_{u v}=\mathrm{F}_{u}\left(W_{v}\right) \subseteq W_{u} \subseteq V_{u}$ and $\Phi_{n, k}[u] \subseteq W_{u}$. The set $\left\{W_{a}: a \in \mathbb{Z}_{n}\right\}$ is a cover of $\mathbb{T}$ iff $r \geq \mathfrak{p}_{n, k}$. In this case $\left\{W_{u a}: u a \in \mathcal{L}\left(\mathbb{W}_{n, k}\right)\right\}$ is a cover of $W_{u}$. The set $\left\{\operatorname{int}\left(W_{a}\right): a \in \mathbb{Z}_{n}\right\}$ is a cover of $\mathbb{T}$ iff $r>\mathfrak{p}_{n, k}$.

Proof: Since $\Phi_{n, k}(v w)=\mathrm{F}_{v}\left(\Phi_{n, k}(w)\right)$ for $v \in \mathbb{Z}_{n}^{*}$ and $w \in \mathbb{W}_{n, k}$, we get $W_{u v}=$ $\mathrm{F}_{u}\left(W_{v}\right)$. Since $\Phi(l(u)), \Phi(r(u)) \in V_{u}$, we get $W_{u} \subseteq V_{u}$. If $a \prec b$ then $\Phi(l(a)) \prec \Phi(l(b))$ since $\Phi(l(b))=\mathrm{R}_{b-a}(\Phi(l(a))$. It follows that $a \prec b$ implies $\Phi(l(u a)) \prec \Phi(l(u b))$. Let $u \in \mathbb{Z}_{n}^{m}$ and $v \in[u]$. Then $\Phi(l(u)) \prec \Phi\left(l\left(v_{[0, m+1)}\right)\right) \prec \Phi\left(l\left(v_{[0, m+2)}\right)\right) \prec \cdots$ Since $\Phi(v)=\lim _{j \rightarrow \infty} \Phi\left(l\left(v_{[0, j)}\right)\right.$, we get $\Phi(l(u)) \prec \Phi(v)$. Similarly we obtain $\Phi(v) \prec \Phi(r(u))$, so $\Phi[u] \subseteq W_{u}$ and we get $W_{u v} \subseteq W_{u}$. For $v=r(0)_{[0, n)}$, the stable fixed point of $\mathrm{F}_{v}$ is

$$
x=\frac{r}{2 \sin \frac{k \pi}{n}}\left[\left(\frac{1}{r}-r\right) \cos \frac{k \pi}{n}+\sqrt{\left(\frac{1}{r}+r\right)^{2} \cos ^{2} \frac{k \pi}{n}-4}\right] .
$$

The intervals $W_{0}$ and $W_{1}$ intersect iff $\Phi(l(1)) \leq \Phi(r(0))$ iff $R_{1}(-x) \leq x$. This means $-x \cos \frac{\pi}{n}+\sin \frac{\pi}{n} \leq x\left(x \sin \frac{\pi}{n}+\cos \frac{\pi}{n}\right)$ or $x \geq \tan \frac{\pi}{2 n}$. This inequality is equivalent to $r \geq \mathfrak{p}_{n, k}$. When this condition is satisfied we get by rotational symmetry that $\left\{W_{a}: a \in \mathbb{Z}_{n}\right\}$ is a cover of $\mathbb{T}$. Similarly, $r>\mathfrak{p}_{n, k}$ iff $\left\{\operatorname{int}\left(W_{a}\right): a \in \mathbb{Z}_{n}\right\}$ is a cover of $\mathbb{T}$. Since $W_{u v}=\mathrm{F}_{u}\left(W_{v}\right)$, we get that $\left\{W_{u a}: u a \in \mathcal{L}\left(\mathbb{W}_{n, k}\right)\right\}$ is a cover of $W_{u}$ provided $r \geq \mathfrak{p}_{n, k}$ and that $\left\{\operatorname{int}\left(W_{u a}\right): u a \in \mathcal{L}\left(\mathbb{W}_{n, k}\right)\right\}$ is a cover of $\operatorname{int}\left(W_{u}\right)$ iff $r>\mathfrak{p}_{n, k}$.

Theorem 13 Let $1 \leq k \leq \frac{n}{2}-1$ and let $\mathrm{F}: \mathbb{Z}_{n}^{*} \times \mathbb{T} \rightarrow \mathbb{T}$ be a hyperbolic number system with quotient $r \leq \mathfrak{q}_{n, k}$. Then $\Phi_{n, k}: \mathbb{W}_{n, k} \rightarrow \mathbb{T}$ is surjective iff $r \geq \mathfrak{p}_{n, k}$ and has the extension property iff $r>\mathfrak{p}_{n, k}$. 
Proof: Assume that $r \geq \mathfrak{p}_{n, k}$ and let $t_{0} \in \mathbb{T}$. There exists $u_{0} \in \mathbb{Z}_{n}$ such that $t \in W_{u_{0}}$. There exists $u_{1}$ such that $u_{[0,1]} \in \mathcal{L}\left(\mathbb{W}_{n, k}\right)$ and $t_{1}:=\mathrm{F}_{u_{0}}^{-1}\left(t_{0}\right) \in W_{u_{1}}$. Continuing in this manner we get a sequence $t_{j}:=\mathrm{F}_{u_{[0, j)}}^{-1}\left(t_{0}\right) \in W_{u_{j}}$ and $\mathrm{F}_{u_{[0, j)}}\left(t_{j}\right)=t_{0}$. If $I \ni t_{0}$, then for all sufficiently large $j$ we have $\left|\left(\mathrm{F}_{u_{[0, j-1)}} \ell\right)(I)\right| \geq 2 \alpha_{r}$, so $\lim _{j \rightarrow \infty} \mathrm{F}_{u_{[0, j-1)}} \ell=\delta_{t_{0}}$ by Lemma 4. Conversely assume that $r<\mathfrak{p}_{n, k}$. Then $\left\{W_{a}: a \in \mathbb{Z}_{n}\right\}$ does not cover $\mathbb{T}$ and therefore $\left\{\Phi[a]: a \in \mathbb{Z}_{n}\right\}$ does not cover $\mathbb{T}$, so $\Phi$ is not surjective. We show that $\Phi_{n, k}: \mathbb{W}_{n, k} \rightarrow \mathbb{T}$ has the extension property provided $r>\mathfrak{p}_{n, k}$. Let $\varphi: \mathbb{W}_{n, k} \rightarrow \mathbb{T}$ be continuous and $u \in \mathbb{W}_{n, k}$. There exists $j_{0}$ and $v_{0}$ such that $\varphi\left(\left[u_{\left[0, j_{0}\right)}\right]\right) \subseteq \operatorname{int}\left(W_{v_{0}}\right)$. There exists $j_{1}$ and $v_{1}$ such that $v_{[0,1]} \in \mathcal{L}\left(\mathbb{W}_{n, k}\right)$ and $\varphi\left(\left[u_{\left[0, j_{1}\right)}\right]\right) \subseteq \operatorname{int}\left(W_{v_{[0,1]}}\right)$. Continuing in this manner, we construct $F(u)=v \in \mathbb{W}_{n, k}$ such that $\Phi_{n, k} F(u)=\varphi(u)$.

$\square$

Corollary 14 Let $1 \leq k \leq \frac{n}{2}-1$ and let $\mathrm{F}: \mathbb{Z}_{n}^{*} \times \mathbb{T} \rightarrow \mathbb{T}$ be a hyperbolic number system with quotient $0<r<1$. Then there exists a factor map $\Phi_{n, k}: \mathbb{W}_{n, k} \rightarrow \mathbb{T}$ such that $\Phi_{n, k}(u)=\arg \lim _{j \rightarrow \infty} \mathbf{F}_{u_{[0, j)}}(0)$ iff $\mathfrak{p}_{n, k} \leq r \leq \mathfrak{q}_{n, k} . \Phi_{n, k}$ has the extension property iff $r>\mathfrak{p}_{n, k}$.

\section{Groups of hyperbolic number systems}

When $\mathrm{F}: \mathbb{Z}_{n}^{*} \times \mathbb{T} \rightarrow \mathbb{T}$ is a hyperbolic number system with $n$ even, then $\mathrm{F}_{a}^{-1}=\mathrm{F}_{a+n / 2}$, so $\mathbb{G}_{n, r}:=\left\{\mathbf{F}_{u}: u \in \mathbb{Z}_{n}^{*}\right\}$ is a countable subgroup of $\mathbb{M}_{\mathbb{D}}$ which we call a group of hyperbolic number system.

Given a subgroup $\Gamma \subseteq \mathbb{M}_{\mathbb{D}}$, the orbit of $z \in \overline{\mathbb{D}}$ is $\Gamma(z)=\{\mathbf{M}(z): \mathbf{M} \in \Gamma\}$. A subgroup $\Gamma \subseteq \mathbb{M}_{\mathbb{D}}$ is called elementary, if there exists $z \in \partial \mathbb{D}$ with finite $\Gamma$-orbit. A subgroup $\Gamma \subseteq \mathbb{M}_{\mathbb{D}}$ is discrete, if the identity (and therefore every $\mathbf{M} \in \Gamma$ ) is an isolated point in $\Gamma$. Some of the groups $\mathbb{G}_{n, r}$ are discrete. This happens for example when $n \geq 8$ and $r^{2}=\left(\cos \alpha_{n}-\sqrt{2 \cos \alpha_{n}-1}\right) /\left(1-\cos \alpha_{n}\right)$, where $\alpha_{n}=\frac{2 \pi}{n}$ (cf. Hyperbolic triangle groups in Magnus [9], page 81). In this case we get a tesselation of the hyperbolic plane by equilateral triangles. We are going to prove that some groups of hyperbolic number systems are dense in $\mathbb{M}_{\mathbb{D}}$. As a direct consequence of Theorem 13 we get Proposition 15.

Proposition 15 Let $n \geq 4$ be even and $\mathfrak{p}_{n, k} \leq r \leq \mathfrak{q}_{n, k}$. Then $\mathbb{G}_{n, r}$ is not elementary.

Lemma 16 Let $n \geq 4$, let $\mathbf{M}$ be a disc $M T$ and set $a=|\mathbf{M}(0)|$. For $t \in \mathbb{R}$ define the t-rotation of $\mathbf{M}$ by ${ }^{t} \mathbf{M}(z)=e^{2 \pi t} \cdot \mathbf{M}\left(z \cdot e^{-2 \pi t}\right)$. Then for every $z \in \mathbb{D}$ with $|z|>\frac{1-\sqrt{1-2 a^{2}}}{a} \approx a$ there exists $k \in \mathbb{Z}_{n}$ such that $\left.\right|^{\frac{k}{n}} \mathbf{M}(z)|<| z \mid$.

Proof: We give the idea of a proof for $n=4$. Because of the rotational symmetry we can assume that $\mathbf{M}(0)=-a, \mathbf{M}^{\bullet}(0)=1$, so $\mathbf{M}(z)=\frac{z-a}{-a z+1}$. The condition $|\mathbf{M}(z)|<$ $|z|$ yields $a|z|^{2}-2 \Re(z)+a<0$ which holds in the open disc $\left(x-\frac{1}{a}\right)^{2}+y^{2}<\frac{1-a^{2}}{a^{2}}$. This circle intersects the lines $y= \pm x$ in $x=f(a):=\left(1-\sqrt{1-2 a^{2}}\right) / a$. Thus if $z=x+i y$ and $|z|>f(a)$, we get $|\mathbf{M}(z)|<|z|$ provided $|y|<x,\left.\right|^{\frac{1}{4}} \mathbf{M}(z)|<| z \mid$ provided $|x|<y$, $\left.\right|^{\frac{1}{2}} \mathbf{M}(z)|<| z \mid$ provided $|y|<-x$, and $\left.\right|^{\frac{3}{4}} \mathbf{M}(z)|<| z \mid$ provided $|x|<-y$.

Theorem 17 Let $n \geq 4$ be even and $r<1$. If $\mathbb{G}_{n, r}$ is not discrete, then it is dense in $\mathbb{M}_{\mathbb{D}}$. 
Proof: Let $\varrho$ be the distance corresponding to the Riemannian metric (1) on $T \mathbb{D}$. Given $\mathbf{M} \in \mathbb{M}_{\mathbb{D}}$ and $\varepsilon>0$, we construct an element $\mathbf{F}$ of $\mathbb{G}_{n, r}$ with $\varrho(\mathbf{M}, \mathbf{F})<\varepsilon$. By the assumption there exists a sequence $\mathbf{G}_{m} \in \mathbb{G}_{n, r}$ which converges to the identity. In particular, $\lim _{m \rightarrow \infty} \mathbf{G}_{m}(0)=0$. Using Lemma 16 we can replace each $\mathbf{G}_{m}$ by some its rotation ${ }^{k} \mathbf{G}_{m} \in \mathbb{M}_{\mathbb{D}}$, so that $\lim _{m \rightarrow \infty} \mathbf{G}_{m} \cdots \mathbf{G}_{0} \mathbf{M}(0)=0$. Thus there exists $\mathbf{G} \in \mathbb{G}_{n, r}$ such that $|\mathbf{G M}(0)|<\varepsilon / 4$. Since $\arg \left(\operatorname{Id}^{\bullet}(0)\right)=0$ (we assume $-\pi \leq \arg (z)<\pi)$, there exists $\mathbf{K} \in \mathbb{G}_{n, r}$ with arbitrarily small $\arg \left(\mathbf{K}^{\bullet}(0)\right)$, and for some its iteration we get $\left|\arg \left(\mathbf{K}^{p} \mathbf{G M}\right)^{\bullet}(0)\right|<\varepsilon / 2$, while $\left|\mathbf{K}^{p} \mathbf{G M}(0)\right|<\varepsilon / 2$. This means $\varrho\left(\mathbf{K}^{p} \mathbf{G M}, \mathrm{Id}\right)<\varepsilon$. Since $\varrho$ is preserved by any disc MT, we get $\varrho\left(\mathbf{M}, \mathbf{G}^{-1} \mathbf{K}^{-p}\right)<\varepsilon$.

A non-elementary subgroup $\Gamma \subseteq \mathbb{M}_{\mathbb{D}}$ is discrete iff for each $\mathbf{M}, \mathbf{N} \in \Gamma$, the subgroup $\langle\mathbf{M}, \mathbf{N}\rangle$ generated by $\mathbf{M}$ and $\mathbf{N}$ is discrete (see Katok [5], Theorem 2.4.8). By a theorem of Jørgensen (see Katok [5], Theorem 2.4.6), if $\langle\mathbf{M}, \mathbf{N}\rangle$ is discrete and nonelementary, then $\left|\operatorname{tr}^{2}(\mathbf{M})-4\right|+\left|\operatorname{tr}\left(\mathbf{M N M} \mathbf{M}^{-1} \mathbf{N}^{-1}\right)-2\right| \geq 1$ (the trace of $\mathbf{M N M} \mathbf{M}^{-1} \mathbf{N}^{-1}$ is well-defined). Thus to show that a group $\mathbb{G}_{n, r}$ is not discrete, it suffices to find two its elements which violate the $\mathbf{J} \varnothing$ rgensen inequality.

Proposition 18 For $n=4, r=\mathfrak{p}_{4,1}=\mathfrak{q}_{4,1}=\sqrt{2}-1$, the group $\mathbb{G}_{4, r}$ is discrete.

Proof: We have $\mathbb{G}_{4, r}=\left\{\mathbf{F}_{u}: u \in \mathcal{L}\left(\mathbb{W}_{n, 1}\right)\right\}$ which is discrete by Theorem 10.

Theorem 19 For even $n \geq 6$ and $\mathfrak{p}_{n, 1} \leq r \leq \mathfrak{q}_{n, 1}$, the groups $\mathbb{G}_{n, r}$ are dense in $\mathbb{M}_{\mathbb{D}}$.

Proof: For $n=6$, a pair of elements $\mathbf{M}=\mathbf{F}_{024}, \mathbf{N}=\mathbf{F}_{042}$ violates the Jørgensen inequality. As $r$ goes from $\mathfrak{p}_{6,1}$ to $\mathfrak{q}_{6,1}$, the value of $\operatorname{tr}^{2}(\mathbf{M})$ goes from 3.375 to 3.704 . The value of $\operatorname{tr}\left(\mathbf{M N M}^{-1} \mathbf{N}^{-1}\right)$ goes from 2.094 to 2.012. For $n=8$, the pair of elements $\mathbf{M}=\mathbf{F}_{025}, \mathbf{N}=\mathbf{F}_{035}$ violates the Jørgensen inequality. For $n \geq 10$, the pair of elements $\mathbf{M}=\mathbf{F}_{0, \frac{n}{2}-1}, \mathbf{N}=\mathbf{F}_{0, \frac{n}{2}+1}$ violates the Jørgensen inequality.

\section{Representation of Möbius transformations}

For every $u \in \mathbb{Z}_{n}^{*}$ and $v \in \mathbb{W}_{n, k}$, we have $u v \in \mathbb{X}_{n, r}$ and $\Phi_{n, k}(u v)=\mathrm{F}_{u}\left(\Phi_{n, k}(v)\right)$. For each $u \in \mathbb{Z}_{n}^{*}$, the transformation $\mathrm{F}_{u}: \mathbb{T} \rightarrow \mathbb{T}$ has symbolic representation $\widetilde{F_{u}}: \mathbb{X}_{n, r} \rightarrow \mathbb{X}_{n, r}$ given by $\widetilde{F_{u}}(v)=u v$, since $\Phi_{n, k} \widetilde{F_{u}}=\mathrm{F}_{u} \Phi_{n, k}$.

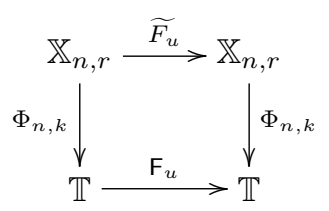

If $\mathbb{G}_{n, r}$ is dense in $\mathbb{M}_{\mathbb{D}}$, then a transformation $\mathrm{M} \in \mathbb{M}_{\mathbb{T}}$ can be represented in the alphabet $A=\mathbb{Z}_{n} \cup\{$.$\} as a left-infinite word v=\cdots v_{-3} \cdot v_{-2} . v_{-1}$, where $v_{-j} \in \mathbb{Z}_{n}^{+}$, such that $\lim _{j \rightarrow \infty} \mathrm{F}_{v_{-j}} \circ \cdots \circ \mathrm{F}_{v_{-1}}=\mathbf{M}$ in $\mathbb{M}_{\mathbb{T}}$. Then for each $u \in \mathbb{W}_{n, k}$ we have

$$
\delta_{\mathrm{M}\left(\Phi_{n, k}(u)\right)}=\lim _{j \rightarrow \infty} \mathrm{F}_{v_{-j}} \cdots \mathrm{F}_{v_{-1}} \mathrm{~F}_{u_{[0, j)}} \ell
$$


A quite interesting use of these symbolic representations would be construction of efficient algorithms for arithmetical operations. Hyperbolic number systems could avoid the overflow problems of computer arithmetics, since $\infty$ is a legitimate number in the system. At least two arithmetical operations are trivial in hyperbolic number systems. To obtain the negation of a number, just interchange $a$ with $n-a$. To obtain the inverse element, interchange $a$ with $\frac{n}{2}-a$ (provided $n$ is even). Other arithmetical operations are less obvious, but they can be described and implemented by passing through standard real arithmetic. Of course, their direct combinatorial description would be much more interesting.

\section{Acknowledgments}

The research was partially supported by the Research Program CTS MSM 0021620845. A part of this research has been done during my visit at Centre de Physique Théorique de CNRS, Luminy, Marseille. A preliminary version of the paper appeared in [7].

\section{References}

[1] M. F. Barnsley. Fractals everywhere. Morgan Kaufmann Pub., 1993.

[2] A. Cauchy. Sur les moyens d'éviter les erreurs dans les calculs numeriques. Comptes Rendus de l'Academie des Sciences, 11:789-798, 1840.

[3] G. A. Edgar. Measure, Topology, and Fractal Geometry. Undergraduate Texts in Mathematics. Springer-Verlag, Berlin, 1990.

[4] C. Frougny. Non-standard number representation: computer arithmetics, beta-numeration and quasi-crystals. In J.P.Gazeau, J.Nešetřil, and B.Rovan, editors, Physics and Theoretical Computer science. IOS Press, 2007.

[5] S. Katok. Fuchsian Groups. Chicago Lectures in Mathematics. The University of Chicago Press, Chicago, 1992.

[6] P. Kůrka. Topological and symbolic dynamics, volume 11 of Cours spécialisés. Société Mathématique de France, Paris, 2003.

[7] P. Kůrka. Octahedron: a holomorphic symbolic representation of the complex sphere. Technical Report CTS-07-15, Center for Theoretical Study, Prague, 2007.

[8] D. Lind and B. Marcus. An Introduction to Symbolic Dynamics and Coding. Cambridge University Press, Cambridge, 1995.

[9] W. Magnus. Noneuclidean tesselations and their groups. Academic Press, New York, 1974.

[10] K. Weihrauch. Computability. EATCS Monographs on Theoretical Computer Science. SpringerVerlag, Berlin, 1987. 